\title{
A Cosmological Scaling Relation for Describing the Late Time Dynamics
}

\author{
Gerardo Cristofano $^{1,2}$ and Orlando Luongo ${ }^{1,2,3}$ \\ ${ }^{1}$ Dipartimento di Fisica, Università di Napoli "Federico II", Via Cinthia, I-80126 Napoli, Italy \\ ${ }^{2}$ INFN Sez. di Napoli, Compl. Univ. Monte S. Angelo Ed. N Via Cinthia, I-80126 Napoli, Italy \\ ${ }^{3}$ Instituto de Ciencias Nucleares, Universidad Nacional Autónoma de México, AP 70543, 04510 México, DF, Mexico
}

Correspondence should be addressed to Orlando Luongo; luongo@na.infn.it

Received 24 March 2013; Revised 16 June 2013; Accepted 26 June 2013

Academic Editor: Rong-Gen Cai

Copyright (C) 2013 G. Cristofano and O. Luongo. This is an open access article distributed under the Creative Commons Attribution License, which permits unrestricted use, distribution, and reproduction in any medium, provided the original work is properly cited.

\begin{abstract}
A scaling relation between mass and minimal information of a given system is inferred from primordial black holes. Extending its validity, it is possible to describe different stages of the universe evolution. Particularly, the broad interest on matching the scaling law, from early to late redshift regimes, may suggest the mechanism to relate quantum and classical aspects of gravity. Under this hypothesis, the scaling relation is interpreted as a thermodynamic modification able to describe the cosmological dynamics at late times. In this scheme, dark energy emerges, as a consequence of assuming the validity of our scale relation. The corresponding equation of state reduces to a cosmological constant at early times and evolves in terms of the apparent horizon at late times.
\end{abstract}

\section{Introduction}

The challenge of reproducing the observed scales, from small to high structures, has been extensively investigated in the last decades [1]. It follows from the particular idea that self-gravitating aggregated structures may be predicted from prime principles [2]. This leads to intrinsic connections between quantum mechanics and gravity, providing a unified scheme for quantum gravity. A similar approach has been already employed several decades ago. In particular, it was found a possible correspondence between proton and higher sizes structure masses [3-5]. Even though this fact may represent a first step for obtaining a self-comprehensive theory, including quantum effects in gravitation, the strange correspondence between proton and heavier masses is not yet clarified [6]. The corresponding problem is known in the literature as coincidence problem [7-10]. All the suggestions and attempts spent to describe the latter coincidence appeared to be nonconclusive in the framework of astrophysics. However, more recently, an accurate explanation has been proposed, dealing with a possible quantization relation for physical systems $[11,12]$. If one assumes black holes as physical systems, it follows that the main interest of the coincidence problem lies on the possible connection of macroscopic scales to the Planck constant $\hbar$ [13]. In doing so, under the hypothesis that the early time phases of the universe evolution could be characterized by primordial black holes, one may infer the observed structures as determined from a self comprehensive common origin, depending on quantum fluctuations [14]. The importance of investigating the consequences of a new quantization rule is to frame the large scale structures into a unifying theory. To this end, it has been shown [15, 16] that it is possible to get a quantization relation which has the meaning of scaling relation between mass and the quantization number.

In this work, by supposing its validity, we describe the consequences of it, at higher and smaller sizes, showing the role played by quantum fluctuations and the corresponding consequences at late time epochs. The observed dynamics today could be influenced by such conditions. We therefore show that it is possible to get a characteristic length of gravitational interaction, showing dark energy effects from prime principles [17]. Moreover, we describe in detail some thermodynamic consequences of our quantization rule, showing that both entropy and temperature become functions of the quantizing number, hereafter $n$. In doing so, it 
becomes evident that our scaling relation, although derived at Planck scales, can be used for all self-gravitating astrophysical systems, including the whole universe.

The paper is organized as follows. In Section 2 we describe in detail the main features of the quantization relation. In Section 3 we extend the quantization law from minimal physical regions to higher ones and we give emphasis to the form of entropy and temperature. In Section 4 we highlight a corresponding cosmological application of our framework, which is able to predict the dark energy effects at late times, without the need of introducing any further cosmological fluid. Finally, Section 5 is devoted to conclusions and perspectives of our work.

\section{The Quantization Relation}

In this section, we describe the main steps leading to the quantization relation, derived from a black hole effective potential, of the early phases of the universe evolution. In this picture, one may assume that primordial black holes dominated the whole universe dynamics $[18,19]$. In doing so, the corresponding scalar potential is

$$
V_{\mathrm{BH}}=e^{2 \phi}\left(Q_{e}-a Q_{m}\right)^{2}+e^{-2 \phi} Q_{m}^{2},
$$

where $Q_{e}$ and $Q_{m}$ represent the electric and magnetic charges, respectively. The so-called dilaton field is defined by the function $\phi$, while the axion field is here represented by $a$.

This framework is restricted to the so-called extremal black holes, in which the event horizons degenerate. It is worth noticing to focus on this case, following the work of $[11,12]$. Let us consider

$$
V_{\mathrm{eff}}^{\mathrm{CFT}}=R_{c}^{2}\left(Q_{e}-\frac{\theta}{2 \pi} Q_{m}\right)^{2}+\frac{1}{R_{c}^{2}} Q_{m}^{2},
$$

where $R_{c}$ is the compactification radius of the so-called Fubini scalar field. Once the effective potential is defined, it is easy to get the following identifications:

$$
R_{c}^{2}=e^{2 \phi}, \quad \frac{\theta}{2 \pi}=a .
$$

Having considered one component charge in (1), stability requirements lead to $a=\theta / 2 \pi=0$, which describes the case in which only the dilaton field is present, giving as the black hole potential the following simplified expression [20]:

$$
V_{\mathrm{BH}}=e^{2 \phi} Q_{e}^{2}+e^{-2 \phi} Q_{m}^{2} \text {. }
$$

Furthermore, by considering $\phi$ as a constant field the following relation between black hole mass and effective potential gets implemented [21]:

$$
G M^{2}=V_{\mathrm{BH}} \cdot
$$

By imposing the stability $\partial V_{\mathrm{BH}} / \partial \phi=0$, one gets

$$
e^{2 \phi_{H}}=\frac{Q_{m}}{Q_{e}}=R_{H}^{2},
$$

with $\phi_{H}$ and $R_{H}$ referring to as their values at the horizon of the black hole. By recasting the mass in terms of the product of the two charges, that is, $G M^{2}=2 Q_{e} Q_{m}$, and assuming the Dirac quantization condition $2 Q_{e} Q_{m}=n \hbar c$, with $n$ a positive integer, we finally get

$$
G M^{2}=n \hbar c .
$$

For $n=1$, we obtain the lowest mass allowed for a quantum black hole (primordial black hole) $M_{\mathrm{BH}} \equiv M_{\text {Planck }}$.

Here, our main purpose is to relate thermodynamics to (7). In doing so, we show that the physical meaning of $n$, if it becomes a function of the size of the system, that is, $\mathscr{R}=$ $\mathscr{R}(t)$, becomes that of an information parameter. In other words, by employing $n=n(\mathscr{R})$, as the minimal information of a given space time region, it is possible to recover the holographic principle and the Verlinde's recipe [22-26]. This will be highlighted in the next sections.

\section{Extending the Quantization Law to Minimal Physical Regions}

By following the pioneering work of $[11,12]$, we extend the quantizing relation, proposed for primordial black hole dominated era, to different epochs along the universe evolution. In particular, we find that one possibility to relate our formula to higher radii, is to consider a continuous $n$, function of the Hubble radius $\mathscr{R}=\mathscr{R}(t)$, with $t$ the cosmic time. After cumbersome algebra, one is able to depict a model which shows a redshift evolution that predicts, at late time, an accelerating universe under some conditions. The holographic principle could be recovered if $n$ is reviewed as the functional term which describes the minimal information of the space time region under exam.

3.1. The Entropy Representation. The concept of entropy turns out to be very useful to describe the thermodynamical processes which imply an increasing disorder. In astrophysics, all the processes are expected to occur only if the entropy increases as the universe expands. However, that is mainly true for all astrophysical systems. It follows that the use of entropy leads to numerous applications, spanning from virialized systems, to black hole physics. It is commonly believed that the black hole entropy depends on its area, rather than its volume, as in standard thermodynamics. In the context of black hole physics, this turns out to be a natural consequence of the macroscopic horizon, associated to every black hole. The net energy content is assumed to be confined within the horizon itself, and then the corresponding first and second laws of thermodynamics can be easily inferred if one considers the area as $A_{\mathrm{bh}}=4 \pi R_{\mathrm{bh}}^{2}$, with $R_{\mathrm{bh}}$ the black hole radius (In this section, we omit the units $\hbar, c$, and $G$ for the sake of clearness. In the incoming sections we will restore their use.).

It is easy to show that the total mass contained into a black hole is $d M=(\kappa / 8 \pi) d A_{\mathrm{bh}}$, where $\kappa$ is the surface gravity of the black hole which can be computed in a model independent way, showing that it is not necessary to fix a priori the black hole mass through a ruler constant. For our purposes, the 
first law of thermodynamics reads $d E=T d S$, which naturally defines a corresponding black hole temperature

$$
T_{\mathrm{bh}}=\frac{1}{8 \pi M}\left(\frac{\hbar c^{3}}{G k_{B}}\right)
$$

that can be recasted as

$$
T_{\mathrm{bh}}=\frac{\kappa}{2 \pi}
$$

showing a simple connection between the black hole surface gravity and its temperature. After cumbersome algebra, it is possible to define a net entropy of the form $S_{\mathrm{bh}}=(1 / 4) A_{\mathrm{bh}}$, which provides a second law

$$
d S_{\text {bh }} \geq-d S_{\text {matter }}
$$

commonly called the generalized second law. In the next subsection, we adopt the validity of (9) and (10) to relate $n$ to current observable universe, with particular attention to the validity of (10), studying the consequences of introducing an incoming particle into the physical system under interest.

3.2. The Generalized Scaling Relation. To get a scaling independent relation, applicable in modern cosmology, we must fulfill the conditions on entropy, emphasized in the above sections. Particularly, we can assume

$$
G M^{2}=n(\mathscr{R}) \hbar c,
$$

where a functional dependence of $n$ on the radius of black holes has been introduced. Under this hypothesis, we get

$$
2 G M d M=d n \hbar c,
$$

so, recalling (7), we infer the density $\rho \equiv d M / d V$, given by

$$
\rho^{2}=\frac{\hbar c}{G} \frac{d n}{d V^{2}}
$$

where we made use of the fact that $\rho \approx M / V$. Equation (13) differs from the approaches proposed in [27, 28], since our relation has been obtained in terms of a generic black hole radius, $\mathscr{R}$. In other words, since the validity of (11) is general, as one can see in $[11,12]$, our relation corresponds to a quantization rule valid for gravitational quantum systems. For these reasons, we want to demonstrate, in what follows, that the use of our quantization relation, in observational cosmology, may lead to accelerate the universe today, without invoking a priori a dark energy term, as responsible for the cosmic speed up.

Together with (13) we are able to assume that

$$
d M=\frac{\lambda_{c}^{M}}{R_{S}^{M}} M d n
$$

in which we used the definitions of Compton length and Schwarzschild radius, that is, $\lambda_{c}^{M}$ and $R_{S}^{M}$, respectively. Equation (14) can be rewritten as

$$
k_{B} T=\frac{1}{2 \pi} \frac{\hbar}{c} \frac{G M}{R_{S}^{2}},
$$

and together with the quantization relation, we have

$$
k_{B} T=\frac{1}{8 \pi} \frac{M c^{2}}{n}
$$

and then one infers

$$
\frac{S}{k_{B}}=4 \pi n
$$

The entropy of a given region of space time is therefore associated to $n(\mathscr{R})$. By combining $N=4 \pi n$ and (17) with the expression for the degrees of freedom, one gets

$$
N k_{B} T=\frac{1}{2} M c^{2}
$$

which relates the temperature to the mass of the universe. Since the Unruh temperature is recovered under our picture, it is easy to notice that our quantized rule appears to satisfy the basic demands of thermodynamics.

3.3. Information of Incoming Particles. From another point of view, one can wonder whether the information due to the introduction of $n(\mathscr{R})$ allows us to recover the first principle of thermodynamics, if one adds energy to the system. As a toy model, let us assume the simplest case of one black hole. By assuming that $n$ scales with respect to the radius of our black hole we get

$$
T d S=\frac{1}{8 \pi} \frac{\hbar c^{3}}{G M k_{B}} 4 \pi d n k_{B}=\frac{1}{2} \frac{M c^{2}}{n} d n,
$$

which represents the first principle in terms of the entropy $S$. Easily we have $d n=2(G M d M / \hbar c)$. So that, by substituting the definition of $n$ into (19)

$$
T d S=2 F_{N} R_{S}
$$

we define $F_{N}$ as the standard Newtonian law. Equation (20) is compatible with the Newtonian dependence on the radius $\mathscr{R}$, because it reproduces the Uhruh law without corrections; that is,

$$
T d S=2(d M) R_{S},
$$

giving

$$
d S=4 \pi k_{B} d M \cdot \frac{2 \pi c}{\hbar} R_{S}=4 \pi k_{B} \frac{R_{S}^{M}}{\lambda_{c}^{M}},
$$

which is compatible with (17) for the definition, $R_{S}^{M}=d n \lambda_{c}^{M}$. On the other side, for an incoming particle, assuming that the volume of a $\mathrm{BH}$ is fixed,

$$
d U=d M c^{2}
$$

therefore, noticing that

$$
d U=c^{2} \frac{\hbar c}{2 G M}=\frac{d n}{2} \frac{\hbar c}{G M^{2}} M c^{2}=\frac{1}{2} M c^{2} \frac{d n}{n}
$$


that reproduces the first principle $d U=T d S$ when one takes the fixed volume evolution. Moreover, the identity holds $F_{N} R_{S}=U / 2=(1 / 2) M c^{2}$. The origin of the factor $1 / 2$ arises because we are considering a fixed mass $M$.

In other words, by evaluating the integral $-\int_{R_{S}}^{0}\left(G M(\mathscr{R}) d M / \mathscr{R}^{2}\right) d \mathscr{R}$, with $M=\rho \cdot(4 / 3) \pi \mathscr{R}^{3}$, we cannot reproduce the exact expression for the Newtonian law, which is instead recovered if

$$
M(\mathscr{R})=\rho \cdot 4 \pi \mathscr{R}^{2},
$$

showing that the internal density scales as $\mathscr{R}^{-2}$. Relating $M(\mathscr{R})$ to the $n$ term, it is easy to show that $n$ contains the physical information of a certain system, in analogy with the minimal size identified in the holographic principle.

In other words, by considering the quantizing relation and the fact that one adds physical information to the system, it is possible to reobtain the Newtonian law, if $n$ is associated to the total energy budget.

\section{Connection with Cosmology}

An important consequence arises by assuming that the scale relation could be associated to the net energy budget of the whole universe. In other words, one may consider the universe, as the physical system under exam. So that, by keeping in mind the validity of the anthropic principle, in a Friedmann-Robertson-Walker metric, we consider the apparent horizon, that is, $\mathscr{R} \propto \mathscr{H}^{-1}[29,30]$, as physical radius. Hence, for reproducing the corresponding expression for the entropy $S$, we notice that

$$
n \propto \mathscr{R}^{2},
$$

which reproduces the form of the holographic principle in the limiting case in which the apparent horizon corresponds to the universe size, and $n$ to the minimal energy. This represents an important result of our model, since holography seems to be recovered in a simple and concise way, by only postulating the validity of (13), for the whole universe, in which we considered the temperature as given by the standard Hawking radiation, in terms of the apparent horizon. Thus, by assuming that the volume of the universe scales as $V \propto \mathscr{R}^{3}$ $[31,32]$, we obtain

$$
n \propto \frac{1}{\mathscr{H}^{2}}
$$

in which we considered the fact that the apparent horizon is proportional to the inverse square of the Hubble rate. By considering the Friedmann equations,

$$
\mathscr{H}^{2}=\frac{8 \pi G}{3} \rho_{t}, \quad \dot{\mathscr{H}}+\mathscr{H}^{2}=-\frac{4 \pi G}{3}\left(3 P+\rho_{t}\right),
$$

where $\rho_{t}$ and $P$ are, respectively, the total energy budget and pressure of the universe, through the use of the continuity equation: that is,

$$
\frac{d \rho}{d z}=\frac{3(P+\rho)}{1+z} .
$$

We are able to infer the conditions that relate $\rho$ with $n$ and $V$. If $\rho_{t}=\rho_{m, 0}(1+z)^{3}+\rho$, with $\rho_{m, 0}(1+z)^{3}$ the standard pressureless matter term, by postulating that $\rho$ represents the dark energy counterpart and assuming the standard definition of energy in thermodynamics [33], that is, $\rho \equiv n / V$, we find

$$
\rho \propto n^{-1 / 2}
$$

which is equivalent to require $\rho \propto \mathscr{H}$. The corresponding equation of state of the dark species associated to $n$ reads

$$
\omega \equiv \frac{P}{\rho},
$$

and we can rewrite it, as follows:

$$
\omega=-\left[1+\frac{(1+z)}{6} \frac{d \ln n}{d z}\right],
$$

which is actually negative, mimicking the dark energy effects, when the first derivative of $n$ with respect to the redshift $z$ is positive. If $d n / d z>0$, the $n$ parameter should increase as the universe expands, in agreement with the hypothesis that at early times a significative contribution due to $n$ is significative, and the universe is black hole dominated. Moreover, (32) provides a dark energy term, reproducing a late time acceleration, which can be matched with current observations; that is, $-1 \leq \omega<0$, when $1+\omega \propto$ $d \ln V / d z$. In addition, if the volume is negligibly small, at early times, we have $\omega \approx-1$. This turns out to give us an early time cosmological constant contribution. Nevertheless, as $z \rightarrow \infty$, a cosmological constant term does not influence the early pressure perturbations, that is, $\delta P \approx 0$, since matter dominates over dark energy. However, at late times, an evolving dark energy term is expected, since $\omega$ strongly depends on the form of $V$ in terms of $\mathscr{H}$. Hence, our model reduces to a cosmological constant dark energy at early times, and to a late time evolving dark energy.

\section{Conclusions and Perspectives}

In this work, we propose a quantum scaling relation, derived from black hole physics. We postulate that our scaling relation is able to describe the universe dynamics, by considering prime principles only. In particular, it is possible to show that, under the hypothesis that $n$ is not a integer number, but a function of the apparent horizon of the universe, one infers the Newtonian law, in agreement with the first principle of thermodynamics. This is analogous to the Verlinde's recipe in which gravity appears as a derived effect. So that, by extending this result to cosmological scales, one finds the interesting fact that dark energy arises as an emerging effect due to our scaling relation. In other words, from our basic demands, it is easy to show that volume, force, and thermodynamics functions can be reobtained in a simple and compact picture. Our goal is to recover the holographic principle, by postulating that $n \propto \mathscr{R}^{2}$, where $\mathscr{R}$ represents the apparent horizon of the universe. The corresponding dark energy model predicts an evolving equation of state at late times, reducing to a cosmological constant at early times. 
Future efforts will be devoted to constrain the cosmological model with current data and to extend the validity of our scaling relation to different cosmological scales.

\section{References}

[1] A. D. Sakharov, "The initial stage of an expanding universe and the appearance of a nonuniform distribution of matter," Journal of Experimental and Theoretical Physics, vol. 22, no. 1, p. 241, 1966.

[2] V. F. Mukhanov, H. A. Feldman, and R. H. Brandenberger, "Theory of cosmological perturbations," Physics Report, vol. 215, no. 5-6, pp. 203-333, 1992.

[3] F. Calogero, "Cosmic origin of quantization," Physics Letters A, vol. 228, no. 6, pp. 335-346, 1997.

[4] S. Capozziello and S. Funkhouser, "A scaling law for the cosmological constant from a stochastic model for cosmic structures," Modern Physics Letters A, vol. 24, no. 14, p. 1121, 2009.

[5] S. Capozziello and S. Funkhouser, "Fractal large-scale structure from a stochastic scaling law model," Modern Physics Letters A, vol. 24, no. 22, p. 1743, 2009.

[6] P. M. A. Dirac, "Quantised singularities in the electromagnetic field," Proceedings of the Royal Society A, vol. 133, p. 60, 1931.

[7] P. A. M. Dirac, "A new basis for cosmology," Proceedings of the Royal Society A, vol. 165, p. 199, 1938.

[8] P. A. M. Dirac, “The cosmological constants," Nature, vol. 139, no. 3512, p. 323, 1937.

[9] P. A. M. Dirac, "Cosmological models and the large numbers hypothesis," Proceedings of the Royal Society A, vol. 338, p. 439, 1974.

[10] A. S. Eddington, "Preliminary note on the masses of the electron, the proton, and the universe," Mathematical Proceedings of the Cambridge Philosophical Society, vol. 27, p. 15, 1931.

[11] S. Capozziello, G. Cristofano, and M. De Laurentis, "Primordial black holes, astrophysical systems and the eddington-weinberg relation," Modern Physics Letters A, vol. 26, no. 34, pp. 25492558, 2011.

[12] S. Capozziello, G. Cristofano, and M. de Laurentis, "Astrophysical structures from primordial quantum black holes," European Physical Journal C, vol. 69, no. 1, pp. 293-303, 2010.

[13] S. Capozziello, S. De Martino, S. De Siena, and F. Illuminati, "Phenomenological scaling laws relating the observed galactic dimensions to the microscopic fundamental scales," Modern Physics Letters A, vol. 15, no. 16, pp. 1063-1070, 2000.

[14] J. J. Halliwell, "Introductory lectures on quantum cosmology," in Proceedings of the 1990 Jerusalem Winter School on Quantum Cosmology and Baby Universes, S. Coleman, J. B. Hartle, T. Piran, and S. Weinberg, Eds., World Scientinc, Singapore, 1991.

[15] R. Kallosh, A. Linde, T. Ortín, A. Peet, and A. Van Proeyen, "Supersymmetry as a cosmic censor," Physical Review D, vol. 46, no. 12, pp. 5278-5302, 1992.

[16] S. Fubini, "Vertex operators and quantum Hall effect," Modern Physics Letters A, vol. 6, no. 4, pp. 347-358, 1991.

[17] D. A. Demir, "Vacuum energy as the origin of the gravitational constant," Foundations of Physics, vol. 39, no. 12, pp. 1407-1425, 2009.

[18] C. T. Byrnes, E. J. Copeland, and A. M. Green, "Primordial black holes as a tool for constraining non-gaussianity," Physical Review D, vol. 86, no. 4, Article ID 043512, 9 pages, 2012.
[19] A. G. Polnarev, T. Nakama, and J. Yokoyama, "Self-consistent initial conditions for primordial black hole formation," Journal of Cosmology and Astroparticle Physics, vol. 09, p. 027, 2012.

[20] R. Kallosh, A. Linde, T. Ortín, A. Peet, and A. Van Proeyen, "Supersymmetry as a cosmic censor," Physical Review D, vol. 46, no. 12 , pp. 5278-5302, 1992.

[21] S. Ferrara, K. Hayakawa, and A. Marrani, "Lectures on attractors and black holes," Fortschritte der Physik. Progress of Physics, vol. 56, no. 10, pp. 993-1046, 2008.

[22] S. Roy, Statistical Geometry and Applications to Micro-Physics and Cosmology, Kluwer, 1998.

[23] R. Bousso and R. Harnik, "Entropic landscape," Physical Review $D$, vol. 82, no. 12, Article ID 123523, 19 pages, 2010.

[24] R. Bousso, "Holographic probabilities in eternal inflation," Physical Review Letters, vol. 97, no. 19, Article ID 191302, 4 pages, 2006.

[25] E. P. Verlinde, "On the origin of gravity and the laws of newton," Journal of High Energy Physics, vol. 2011, article 29, 2011.

[26] R. Bousso, "The holographic principle," Reviews of Modern Physics, vol. 74, no. 3, pp. 825-874, 2002.

[27] S. Hod, "Bohr's correspondence principle and the area spectrum of quantum black holes," Physical Review Letters, vol. 81, no. 20, pp. 4293-4296, 1998.

[28] L. Liu and S. Y. Pei, "Sommerfeld's quantum condition of action and the spectra of quantum schwarzschild black hole," Chinese Physics Letters, vol. 21, no. 10, pp. 1887-1889, 2004.

[29] K. Bamba, S. Capozziello, S. Nojiri, and S. D. Odintsov, "Dark energy cosmology: the equivalent description via different theoretical models and cosmography tests," Astrophysics and Space Science , vol. 342, no. 1, pp. 155-228, 2012.

[30] S. Capozziello and M. de Laurentis, "Extended theories of gravity," Physics Reports, vol. 509, no. 4-5, pp. 167-321, 2011.

[31] O. Luongo and H. Quevedo, "Cosmographic study of the universe's specific heat: a landscape for Cosmology?" In press, http://arxiv.org/abs/1211.0626.

[32] R.-G. Cai and S. P. Kim, "First law of thermodynamics and Friedmann equations of Friedmann-Robertson-Walker universe," Journal of High Energy Physics, no. 02, article 050, 2005.

[33] H. B. Callen, Thermodynamics and an Introduction to Thermostatics, John Wiley and Sons, New York, NY, USA, 1985. 

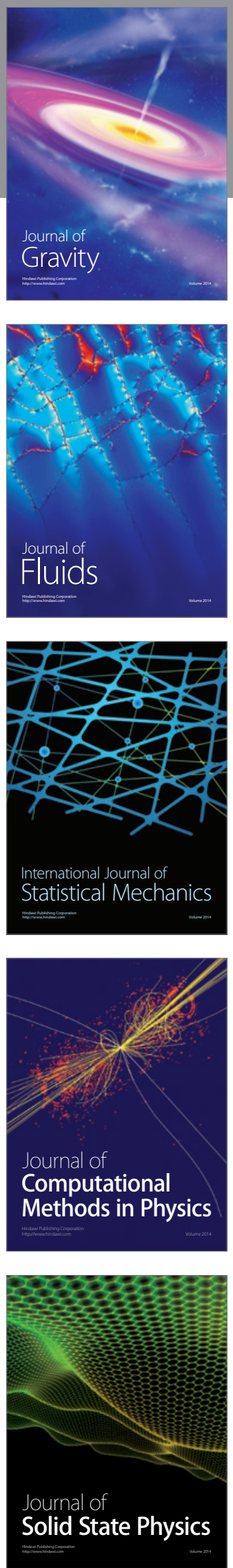

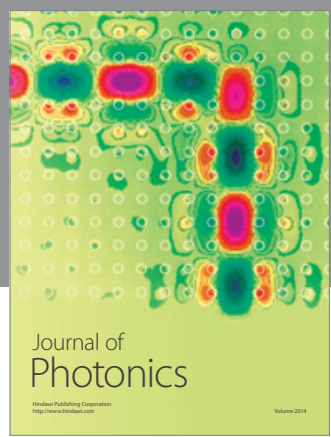

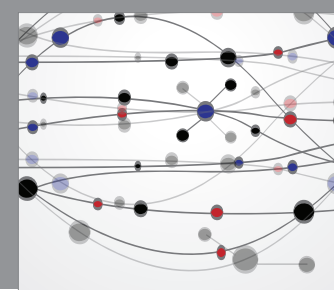

The Scientific World Journal

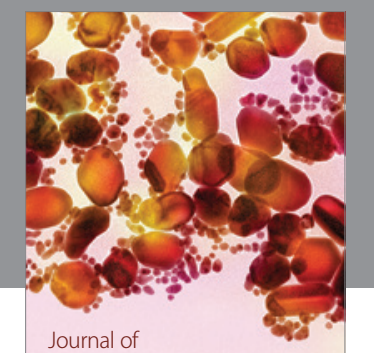

Soft Matter
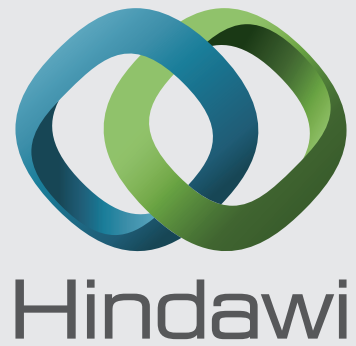

Submit your manuscripts at

http://www.hindawi.com
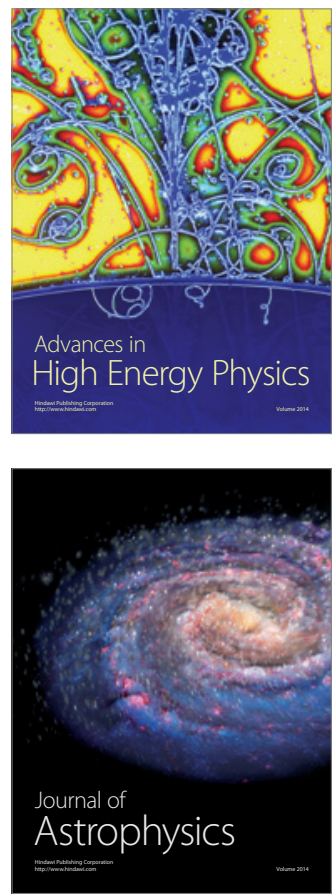
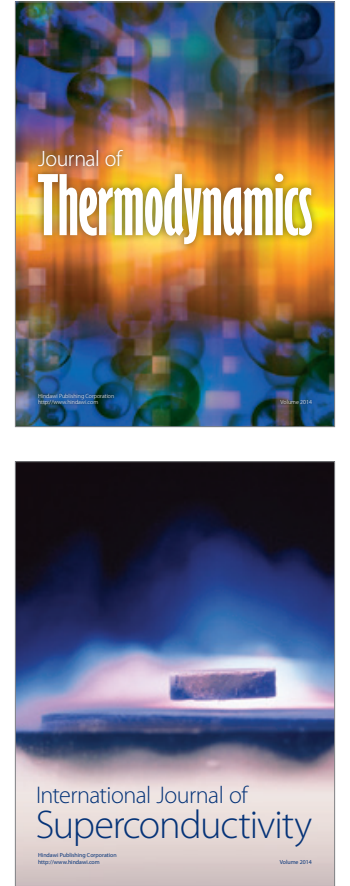
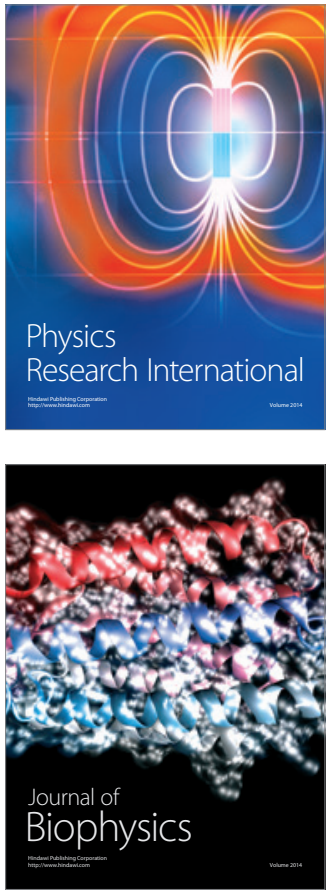
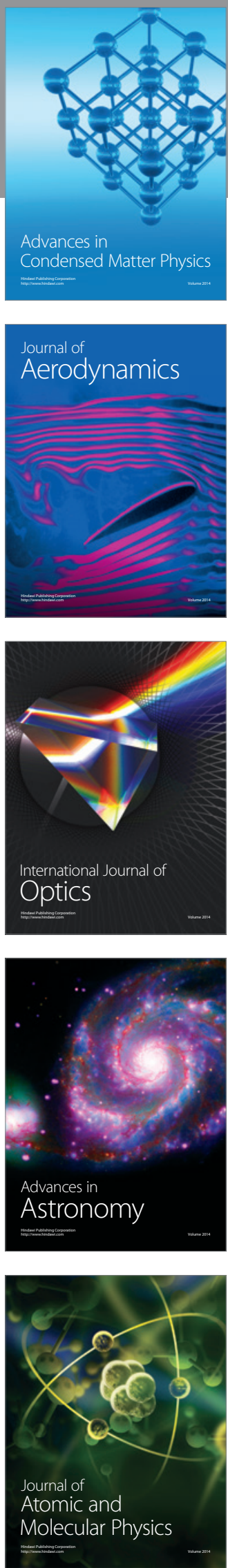